\title{
Half helical turn spacing changes convert a frog into a mouse rDNA promoter: a distant upstream domain determines the helix face of the initiation site
}

\author{
Louise K. Pape, Jolene J. Windle, ${ }^{1}$ and Barbara Sollner-Webb \\ Department of Biological Chemistry, The Johns Hopkins University School of Medicine, Baltimore, Maryland 21205 USA
}

\begin{abstract}
Transcription of frog rDNA by mouse cell factors is the only documented exception to the observed species selectivity of rRNA gene expression. This heterologous transcription is authentic in that it uses the normal frog upstream and core promoter domains, as well as the normal mouse polymerase I transcription factors, but it initiates at residue -4 . We now show that by introducing an insertion or deletion of approximately one-half helical turn anywhere within the 90-bp region between the upstream and core promoter domains, the initiation site moves to residue +1 . Promoters bearing spacing changes of approximately one or two full helix turns do not initiate at residue +1 , whereas a promoter with a one and one-half-turn helical turn spacing change again supports initiation at residue +1 . Thus, the position of the upstream domain of the frog promoter shows a stereo-specific requirement relative to the core promoter domain and dictates the face of the DNA helix on which transcription initiates, 140 bp away. In contrast, relative to the core promoter domain, initiation can occur on either side of the DNA helix. Furthermore, the striking observation that several frog half helical turn spacing change mutants are stronger templates with the mouse factors than the homologous mouse rDNA suggests that the polymerase I transcriptional machinery of even distantly related species is far more similar than generally envisioned.
\end{abstract}

[Key Words: Initiation site; RNA polymerase I; DNA helix; rDNA transcription; species selectivity]

Received May 26, 1989; revised version accepted October 27, 1989.

Eukaryotic RNA polymerase I transcribes a single kind of gene that encodes the precursor to the $18 \mathrm{~S}, 5.8 \mathrm{~S}$, and $28 \mathrm{~S}$ rRNAs. The rDNA promoter region has been mapped and characterized in a number of species, including Xenopus laevis (Moss 1982; Sollner-Webb et al. 1983; Windle and Sollner-Webb 1986a,b; Reeder et al. 1987), mouse (Grummt 1982; Yamamoto et al. 1984; Miller et al. 1985), human (Learned et al. 1983; Smale and Tjian 1985; Haltiner et al. 1986; Jones et al. 1988), and Acanthamoeba (Iida et al. 1985; Kownin et al. 1985). Linker-scanning mutants of both the $X$. laevis and human rRNA genes have demonstrated the presence of two essential promoter regions: an upstream and a core domain (Haltiner et al. 1986; Windle and Sollner-Webb 1986b). In Xenopus, these domains extend from approximately -140 to approximately -128 and from approximately -36 to +10 , respectively. Sequences lying between these two essential domains have a much smaller effect in our assays (Windle and Sollner-Webb 1986b). However, the spacing between the core and upstream domains of the Xenopus rDNA promoter is of critical

1Present address: Regulatory Biology Laboratory, The Salk Institute for Biological Studies, La Jolla, California 92037 USA. importance, as all insertions or deletions of $>2 \mathrm{bp}$ abolish transcription in the Xenopus system /Windle and Sollner-Webb 1986b). The mouse rDNA promoter also consists of an essential core domain extending from approximately -35 to +5 and a stimulatory upstream domain that extends $5^{\prime}$ to approximately residue -150 (Miller et al. 1985). Despite their conserved domain organization, however, the mouse, human, and frog rDNA promoter regions show only very limited sequence homology.

rDNA transcription generally exhibits a marked species selectivity, exemplified by the failure of human rDNA to transcribe in a mouse cell extract, and vice versa (Grummt et al. 1982; Mishima et al. 1982; Miesfeld and Arnheim 1984; Smale and Tjian 1985). Successful heterologous rDNA transcription has typically involved closely related species, for example, mouse/ rat/hamster (Mishima et al. 1982; Vance et al. 1985; Lopata et al. 1986), human/monkey (Learned and Tjian 1982), or X. laevis/X. borealis (Sollner-Webb and McKnight 1982), where the promoter sequences are well-conserved ( $>130$-bp of 150 -bp sequence identity). One notable exception to this paradigm is the specific transcription of Xenopus rDNA in mouse cell extracts 
(Wilkinson and Sollner-Webb 1982). Using 5' deletion mutants of $X$. laevis rDNA, the same large promoter region extending upstream to approximately residue -140 was found to be essential in the heterologous system (Culotta et al. 1987), as in the homologous Xenopus expression systems (Sollner-Webb et al. 1983; Windle and Sollner-Webb 1986a). Furthermore, fractionation studies indicated that the same mouse factors catalyze this heterologous transcription as transcribe the homologous mouse rDNA (Culotta et al. 1987). In fact, the only "unnatural" aspect of this heterologous transcription is that synthesis begins at residue -4 (Wilkinson and SollnerWebb 1982), 4 nucleotides upstream from the site at which $X$. laevis rDNA transcription initiates in vivo and in the Xenopus transcription systems.

The studies described here were initiated to identify the internal organization of the frog rDNA promoter used by the mouse polymerase I machinery. Transcription of Xenopus linker-scanning mutants in the mouse S-100 extract revealed essential upstream and core promoter domains separated by a 90 -bp intervening region of far less rigidly defined sequence, as in the homologous Xenopus system. In the heterologous system, however, mutants that introduce an insertion or deletion of approximately one-half helical turn anywhere throughout the 90-bp interdomain region abolish initiation at the -4 start site and, instead, initiate transcription at residue +1 . The closer these mutations are to an exact half helical turn spacing change, the more efficient their transcription. These data demonstrate a stereo-specific selection of the initiation site by the upstream distal promoter domain. All of the half helical turn spacing change mutants are unexpectedly active, and several of these templates have become "super promoters" that even outcompete the mouse rDNA promoter for its homologous transcription factors. Implications of these results with regard to promoter function and species selectivity are discussed.

\section{Results}

Both the core and upstream promoter domains are required for heterologous $r D N A$ transcription

We demonstrated earlier that cloned $X$. laevis rRNA genes are transcribed in an S-100 extract of mouse tissue culture cells (Wilkinson and Sollner-Webb 1982). This represents the one documented exception to the otherwise high degree of species selectivity observed for transcription of rRNA genes (Grummt et al. 1982; Smale and Tjian 1985) and occurs despite only minimal obvious sequence homology between the mouse and Xenopus rDNA promoter regions. To further define the Xenopus promoter elements recognized by the mouse polymerase I transcription machinery, templates bearing linkerscanning (LS) mutations of the promoter region of the $X$. laevis rRNA genes (Windle and Sollner-Webb 1986b) were assayed for transcriptional capacity in the mouse S-100 extract. Previously, transcription of this series of mutants in Xenopus oocytes revealed two regions essential for directing polymerase I initiation: an upstream domain defined by LS $-140 /-128$ and a core domain defined by LS $-36 /-28$, LS $-18 /-9$, LS $-8 /+1$ and LS $+7 /+10$ (Windle and Sollner-Webb 1986b).

Figure 1A shows the result obtained when the linkerscanning templates were transcribed in the mouse S-100 extract, along with an internal "control" rDNA template. An upstream and a core domain of the $X$. laevis rDNA promoter are seen to be essential in this heterologous mouse extract, akin to the situation in the homologous Xenopus case. In the heterologous system, the upstream domain is defined by LS-140/-128 and LS $-126 /-120$, although quantitatively relevant sequences extend upstream to approximately residue -150 . The core promoter domain for the heterologous transcription is located between residues -29 and +10 , defined by LS $-29 /-22$, LS $-18 /-9$, LS $-8 /+1$, and LS $+7 /+10$ (Fig. 1A).

In addition to the essential core and upstream promoter domains, quantitative effects of the sequences between these two domains are also seen in the mouse cell extract. Most of the linker-scanning mutants with lesions mapping to this region are $10-30 \%$ as active as the wild-type promoter (Fig. 1A), and the X. laevis template LS - 115/-77 (in which 37 bp of this interdomain region was replaced by polylinker sequence; Windle and Sollner-Webb 1986b) is inactive (Fig. 1B). In contrast, when assayed in injected Xenopus oocytes, all of these interdomain mutants were $20-90 \%$ as active as the wild-type gene (Windle and Sollner-Webb 1986b). However, linker-scanning mutants mapping to this region of $X$. laevis rDNA can also appear quite inactive in the homologous Xenopus system when assayed under alternate injection conditions (Reeder et al. 1987), so appreciable effects of mutations in this central region can be observed under heterologous, as well as homologous, transcription conditions.

\section{Half helical turn deletions result in accurate +1 initiations}

Transcription in the homologous Xenopus system is critically dependent on the relative spacing of the upstream and core promoter domains (Windle and SollnerWebb 1986b|, a result demonstrated using derivatives of the linker-scanning mutants in which small insertions or deletions were introduced at the site of the linker. All templates that altered the spacing between the core and upstream domains by $>2$ bp were transcriptionally inactive in the homologous Xenopus system, although their parental linker-scanning constructs were all active (Windle and Sollner-Webb 1986b).

To assess whether this precise spacing requirement was also reflected in the heterologous transcription system, these Xenopus spacing change mutants were used as templates in the mouse cell S-100 extract. The effects of deletion mutants are shown in Figure 2, in lanes alternating with assays of their respective parental linker-scanning mutants. Deletions of $4 \mathrm{bp}$ from LS $-154 /-143$ or LS $+14 /+23$, whose mutations are upstream or downstream of the essential promoter, have no effect on either the level of transcription or the loca- 


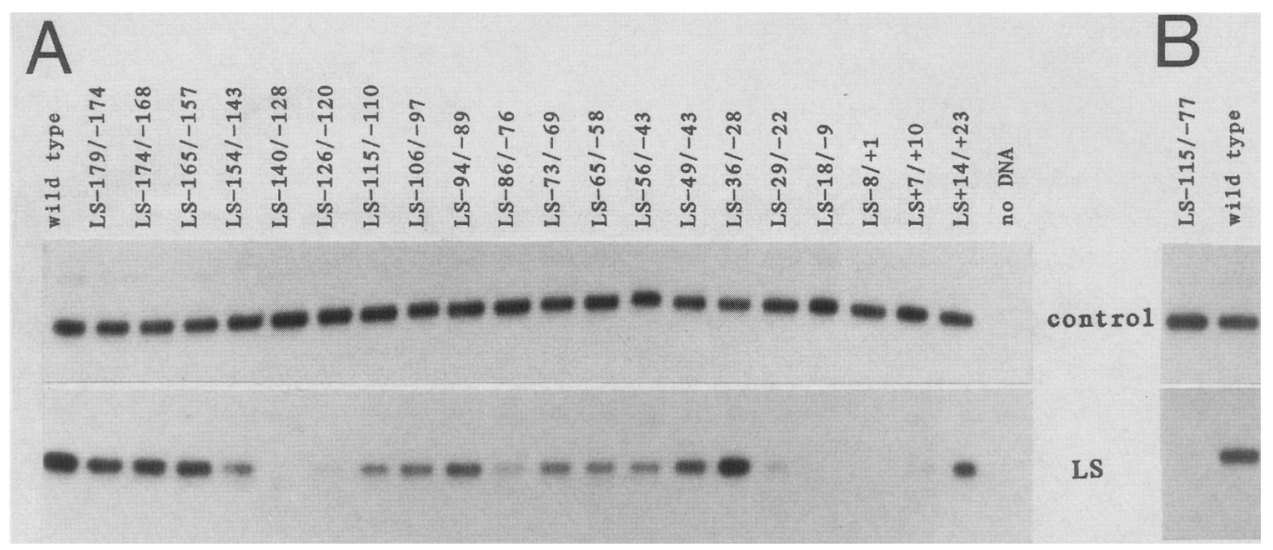

Figure 1. Effect of Xenopus rDNA linker-scanning mutations on transcription in the mouse S-100 extract. $(A)$ Equal amounts of the control $X$. laevis rDNA plasmid and the indicated plasmid bearing either the parental wild-type rDNA or the linker-scanning mutant were cotranscribed in the mouse S-100 extract. The control template contains a wild-type Xenopus rDNA promoter joined upstream of a distinguishable segment of foreign DNA (see Materials and methods). Equal amounts of RNA were hybridized in separate reactions to the probe specific for the transcript of the control gene and the probe specific for the transcript of the wild-type and the linker-scanning templates, and the hybrids were subjected to S1 nuclease analysis. $(B)$ LS $-115 /-77$ and the control gene were assayed as above.

tion of the initiation site, relative to that of the parental template molecule. [Part of the signal from LS $+14 /$ $+23(-4)$ appears as a lower band, as explained in the legend to Fig. 2.] Therefore, as in the homologous Xenopus system, deletions that lie $5^{\prime}$ or $3^{\prime}$ of the essen-

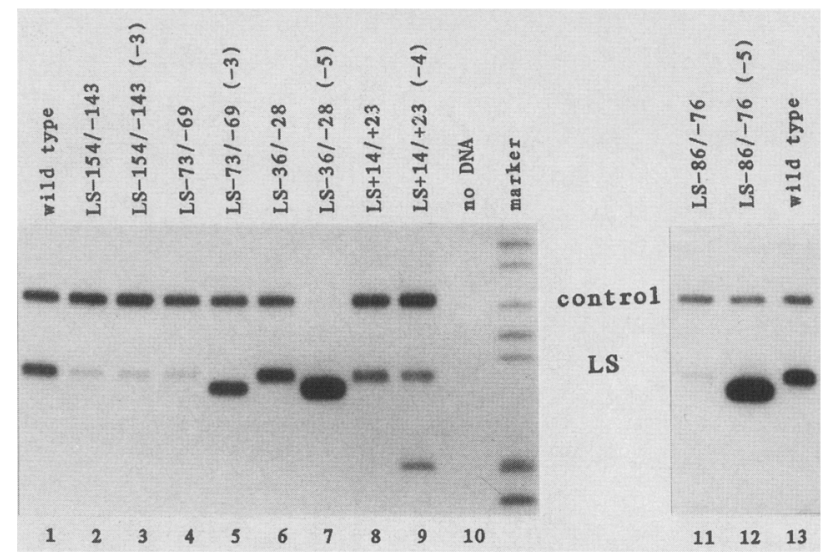

Figure 2. The effect of $\sim 4$-bp deletions on initiation site utilization and transcriptional efficiency in the mouse S-100 extract. Four base pairs were deleted from the linker of the indicated parental linker-scanning mutants, and the resultant deletion mutants were assayed as in Fig. 1A, except that both probes were contained in the same reaction mixture. The spacing changes relative to the wild-type rRNA gene are indicated in parenthesis. The lower band from LS $+14 /+23(-4)$ RNA results from $\mathrm{S} 1$ nuclease cleavage where the probe (which is complementary to the wild-type rRNA sequence) loops out by 4 nucleotides opposite the site of this 4-nucleotide deletion; S1 nuclease does not cleave with LS +14/ + 23 RNA because the probe does not similarly loop out at the site of this linker. Lanes 11-13 are from a different experiment in which the specific activity of the probe assaying the control gene RNA was approximately threefold lower than that assaying the linker-scanning template and the wild-type transcripts. The marker is end-labeled Hpall-cleaved pBR322. tial promoter sequences do not alter the transcriptional signal. Analogous small deletions from LS $-73 /-69$, LS $-36 /-28$, or LS $-86 /-76$, between the core and upstream promoter domains, have a very different effect. As in the homologous case (Windle and Sollner-Webb 1986b), these deletion mutants no longer initiate at the site that is used on the wild-type gene (Fig. 2, lanes 5, 7, and 12). In the mouse extract, however, these deletion templates have the striking property of directing initiation from an altered start site. Notably, the transcriptional efficiency of each of these deletion mutants is dramatically increased relative to their respective parental templates, and both -5-bp mutants are considerably more efficient templates than the -3-bp mutant (Fig. 2, lanes $4-7$ and $11-12$ ).

One notable feature of transcription of Xenopus rDNA in the mouse cell extract is that initiation occurs at position -4 instead of at +1 , the site used in vivo and in the homologous Xenopus expression systems (Wilkinson and Sollner-Webb 1982). To determine the location of the new start site used by the spacing change mutants, an S1 nuclease analysis of their transcripts was resolved on a sequencing gel. As seen in Figure 3 (lanes 7 and 81 , the probe DNA protected by the transcript of LS $-73 /-69(-3)$ precisely comigrates with that protected by authentic $X$. laevis rRNA. Thus, LS-73/ $-69(-3)$ initiates at the normal Xenopus start site, residue +1 . Confirming this conclusion, the $S 1$ nuclease analyses in Figure 3 (lanes 5 and 6), and the sequence analysis of the probe DNA (lanes 1-4) show that the spacing change template initiates 4 nucleotides downstream from residue -4 , the site used by the parental LS - 73/-69 template and by the wild-type Xenopus rDNA in the mouse S-100 extract (Wilkinson and Sollner-Webb 1982). Others of the approximately half helical turn spacing change mutants, including LS $-36 /$ $-28(-5)$, have also been found to initiate at residue +1 




Figure 3. Identification of $X$. laevis initiation sites used in the mouse cell extract. LS $-73 /-69$, LS $-73 /-69(-3)$, and wildtype rDNA $\left(5^{\prime} \Delta-245\right)$ were transcribed in the mouse S-100 extract, as above. In addition, the wild-type rDNA was transcribed in microinjected Xenopus oocytes as described (Windle and Sollner-Webb 1986b). RNA from each was analyzed by S1 nuclease mapping and resolved on a sequencing gel. Under these S1 nuclease conditions, a cluster of protected bands is obtained, the shortest of which represents the 5 ' end of the RNA (Wilkinson et al. 1983). The first four lanes contain a chemical sequence analysis of the probe DNA (Maxam and Gilbert 1980). The insert depicts a graphic representation of the data, incorporating the requisite one and one-half nucleotide correction (Sollner-Webb and Reeder 1979).

in the mouse S-100 extract (not shown). Dinucleotide analysis has further demonstrated that they initiate at the $\mathrm{A}$ residue of the TpApG sequence /residue -1 to +2 ; data not shown). Thus, deleting 4 bp from the rDNA promoter region at residue $-30,-70$, or -80 causes the transcription initiation site to shift from the aberrant "heterologous" -4 position to the authentic in vivo initiation site at residue +1 .
Stereo-specific spacing between the core and upstream domains determines the initiation site

Because deletions within the Xenopus rDNA promoter shift the initiation site downstream, we wished to determine whether insertions would shift the initiation site in the opposite direction. For instance, genes bearing 4-bp promoter insertions might initiate at residue -8 (because residue -8 , like the functional initiation sites at both residues +1 and -4 , begins an AGG motif). To address this, derivatives of the linker-scanning mutants bearing $\sim 4$-bp insertions were assayed in the mouse S-100 extract (Fig. 4). As anticipated, an insertion upstream of the promoter region, LS $-165 /-157(+3)$, had no effect on transcription, and LS $-126 /-120$, in which important sequences of the upstream promoter domain are mutated, was not rescued by the 4-bp insertion. Also, all of the insertions between residues -115 and -28 $[$ LS $-115 /-110(+4), \quad$ LS $-106 /-97(+4), \quad$ LS $-86 /$ $-76(+3), \quad$ LS $-73 /-69(+3), \quad$ LS $-65 /-58(+4)$, and LS $-36 /-28(+3)]$ abolish initiation at the -4 site that is used in the wild-type gene. However, these templates all direct transcription from an alternative position. In addition to the transcript bands readily apparent in Figure 4 (lanes 5-9), longer exposures and other experiments demonstrate that LS $-115 /-110(+4)$ also directs transcription from this alternate start site. Surprisingly, the initiation site of these +3 and +4 insertion mutants is at residue +1 , not residue -8 . Their initiation site was identified by S1 nuclease analysis, as in Figure 3 (data not shown). Thus, transcription initiates at the same +1 position in all of the mutants with the approximately one-half helical turn insertions or the approximately one-half helical turn deletions within the 90-bp region between the core and upstream promoter domains. These results suggest that the upstream promoter domain is critical in determining the initiation site but that it is the relative helical orientation that is important, rather than the precise spacing.

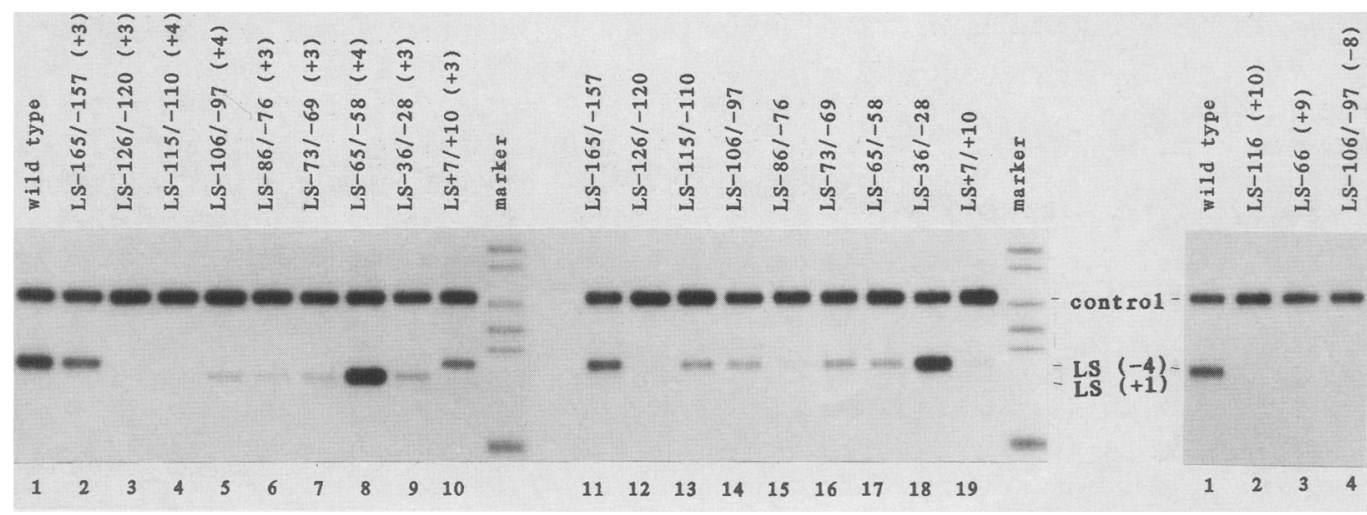

Figure 4. (Left and middle) The effect of $\sim 4-\mathrm{bp}$ insertions on transcription in the mouse S-100 extract. Four-base pair insertions were introduced into the indicated linker-scanning mutants. The insertion mutants, their parental linker-scanning mutants, and the wildtype rDNA were assayed as in Fig. 2. In duplicate experiments in other S-100 extracts, not only does LS $-115 /-110(+4)$ clearly show low levels of +1 initiation, but LS $-126 /-120$ and LS $-126 /-120(+3)$ show perceptible levels of -4 and +1 initiation, respectively.

Figure 5. (Right) The effect of spacing changes of approximately one helical turn on transcription in the mouse cell extract. Ten base pairs were inserted into the wild-type promoter at position -116 , and $9 \mathrm{bp}$ at position -66 ; 8 bp were deleted from LS - 106/ -97 to generate spacing changes approximating one full helix turn. Templates were assayed as in Fig. 2. 

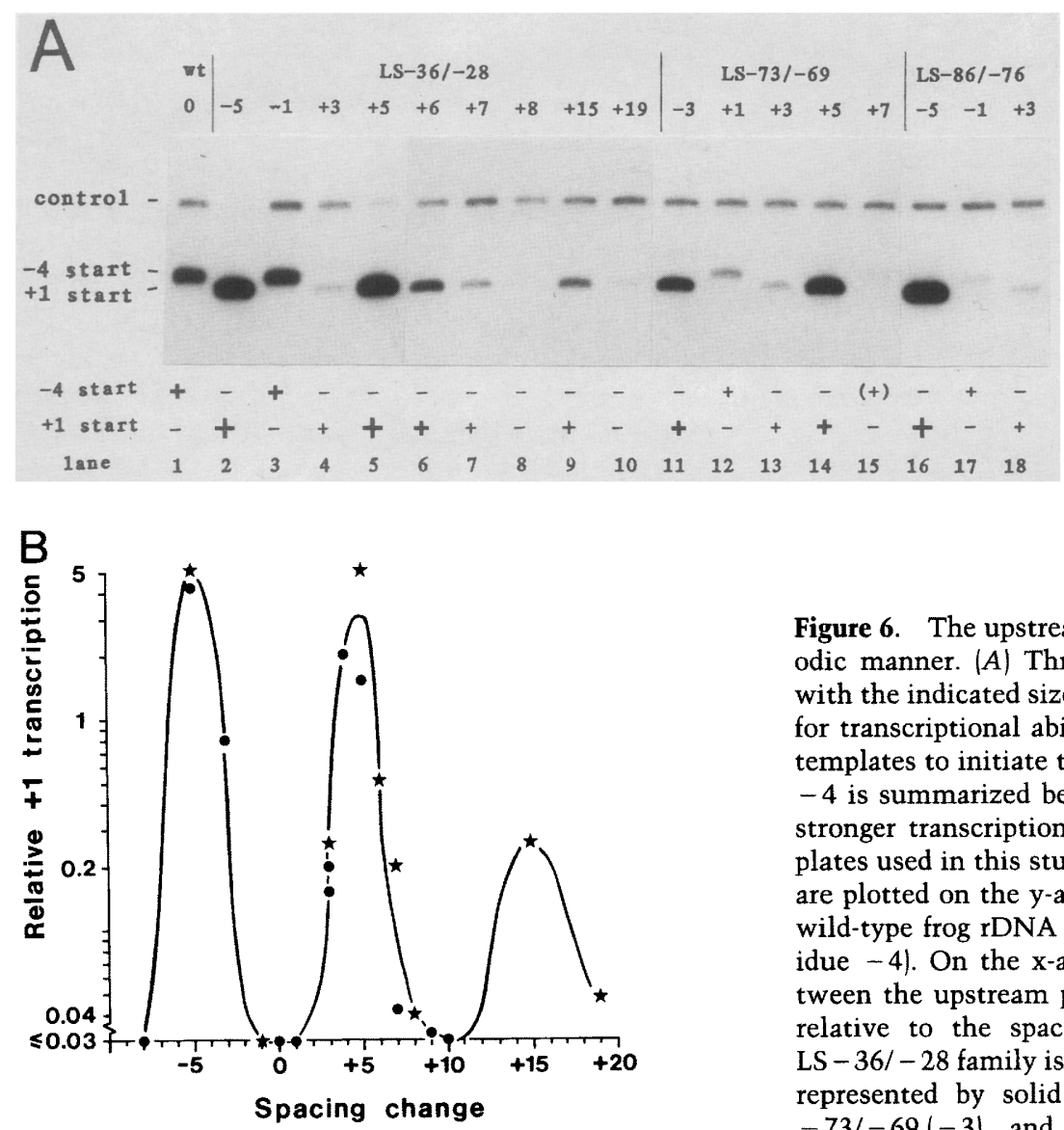

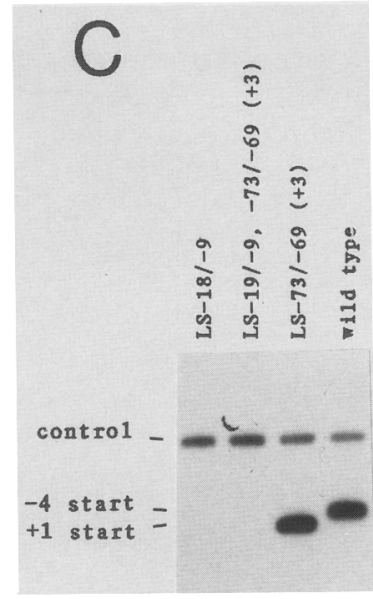

Figure 6. The upstream domain functions in a helix-turn periodic manner. (A) Three families of spacing change mutants, with the indicated sizes of insertions or deletions, were assayed for transcriptional abilities, as in Fig. 2. The capacity of these templates to initiate transcription at residue +1 and at residue -4 is summarized below, with larger plus signs indicative of stronger transcription. $(B)$ The capacities of the various templates used in this study to initiate transcription at residue +1 are plotted on the y-axis /scaled relative to the capacity of the wild-type frog rDNA promoter to initiate transcription at residue -4 ). On the $x$-axis is plotted the change in spacing between the upstream promoter domain and the initiation site, relative to the spacing in the wild-type frog rDNA. The LS $-36 /-28$ family is depicted by stars; the other templates are represented by solid circles. (C) The template LS-18/-9, $-73 /-69(-3)$, and its parental linker-scanning templates were assayed as in $A$. In $A$ and $C$, the probe assaying control gene RNA was approximately threefold lower specific activity than that assaying the transcripts of the LS templates and wild-type gene.

Because all of the mutants bearing insertions or deletions of approximately one-half helical turn anywhere between the upstream and core promoter domains initiate at residue +1 in this heterologous transcription system (Figs. 2-4), we examined the effect of larger length alterations. As shown in Figure 5, templates bearing insertions or deletions of approximately one helical turn at position $-116,-97$, or -66 of the promoter fail to initiate appreciable levels of transcription at any site.

\section{Initiation at residue +1 is responsive to the helical} periodicity of the upstream and core promoter domains

The data of Figures 2-5 strongly suggest that the initiation site is dictated by the relative face of the DNA helix on which the upstream promoter domain is located. The most definitive way to demonstrate this is to study the effect of nested sets of spacing change mutations created at single sites in the promoter. Figure 6A shows the transcription of three such families of spacing change mutants. The LS $-36 /-28$ family is the most extensive (Fig. 6A, lanes $2-10$ ). Correct initiation at residue +1 is not supported by the parental template (a -1 spacing change; lane 3 |, but it is very actively supported by the half helical turn insertion and the half helical turn deletion derivatives (lanes 2 and 5). Notably, insertion mutants of one-third and two-thirds helical turn (lanes 4 and 7) do initiate at +1 but considerably less efficiently than do the true half helical turn insertion mutants, and an intermediate-size $(6-\mathrm{bp})$ insertion mutant initiates at an intermediate efficiency (lane 6). The template bearing close to a full helical turn insertion (the +8 mutant; lane 8 ) barely supports initiation at +1 . Moving to still larger size alterations, the template with a one and onehalf turn insertion (lane 9) again supports +1 initiation, and the template carrying an insertion of approximately two helical turns (the +19 mutant; lane 10) again is virtually inactive. Thus, the capacity of the LS $-36 /-28$ family to initiate correctly at residue +1 is periodic with the number of DNA helical repeats between the upstream and core promoter domains.

Analyses of the spacing change mutants of the LS-73/-69 and LS-86/-76 families further strengthen the conclusion that rDNA transcriptional initiation reflects the helical periodicity of the promoter domains. In the LS $-73 /-69$ family (Fig. 6A, lanes $11-15$ ), the +1 mutant initiates only at residue -4 and not at residue +1 , the -3 and +3 mutants initiate at 
residue +1 but only with moderate efficiency, the +5 mutant initiates very efficiently at residue +1 , and the +7 mutant initiates weakly at residue +1 . Curiously, the +7 mutant also shows some initiation at residue -4 (whereas the $-3,+3$, and +5 mutants do not), indicating that initiation at residue -4 can also exhibit a somewhat periodic spacing requirement. Similarly in the LS $-86 /-76$ family (Fig. 6A, lanes 16-18), the parental -1 deletion template initiates at residue -4 and not at +1 , the -5 mutant initiates very efficiently at residue +1 , and the +3 mutant initiates with considerably lower efficiency at residue +1 .

The abilities of all of the frog templates with mutations between residues -115 and -28 to direct correct initiation at residue +1 in this heterologous mouse cell extract is summarized in Figure 6B. The $\sim 10 \cdot \mathrm{n}$ base pair periodicity is readily apparent, extending over a $30-b p$ variation in spacing between the upstream and core promoter domains. Because the primary sequence within this region has quantitative effects on the level of transcription (Fig. 1), one would expect mutants with equal size spacing changes derived from different parental linker-scanning constructs to exhibit somewhat different levels of transcription, as is observed (Fig. 6B). However, both from the average transcriptional efficiency of the various mutants with each spacing change (the heavy curve in Fig. 6B) and from the absolute transcriptional efficiency of one family of spacing change mutants /the LS -36 / - 28 family members, represented by stars in Fig. 6B), it is clear that the efficiency of transcriptional initiation at +1 exhibits a periodicity according to the relative helical face of the upstream promoter domain.

The data of Figures 2-6 have demonstrated that the side of the DNA helix on which transcription initiates is determined by the helical orientation of the -140 promoter domain. In contrast, the core promoter domain does not show a fixed orientation relative to the initiation site, for it is compatible with initiation both at residues -4 and +1 . To confirm this unanticipated conclusion, it was important to demonstrate that the sequences of the core promoter domain function in directing initiation at residue +1 in the mouse S-100 extract. Thus, we created a double mutant between the spacing change LS $-73 /-69(-3)$ mutant, which is very active at directing +1 initiation, and the LS $-18 /-9$ mutant, which is inactive. Figure $6 \mathrm{C}$ shows that this double mutant fails to initiate transcription at residue +1 , demonstrating that the core promoter domain is important for initiation both at residue +1 and at residue -4 on the opposite face of the DNA helix.

\section{Several frog promoters can outcompete the mouse promoter}

Although the wild-type frog rDNA promoter directs transcription in the heterologous mouse cell extract, the level of expression is considerably lower than that obtained with the homologous mouse rDNA promoter. This is illustrated in Figure 7A, where the mouse rDNA template (lane 3) yields nearly an order of magnitude more transcript than does the wild-type $X$. laevis promoter (lane 1). To examine the relative efficiency of one of the very active frog linker-scanning mutants bearing a half helical turn deletion, LS $-36 /-28(-5)$ was transcribed in a parallel reaction (lane 2). The activity of this template in the mouse S-100 extract is seen to be comparable to that of the homologous mouse promoter.

Next, we examined the frog templates for their abilities to form stable transcription complexes, the longlived associations between initiation factors and promoter sequences that can direct many rounds of transcription (Cizewski and Sollner-Webb 1983). As seen in Figure $7 \mathrm{~A}$, the wild-type frog promoter is inefficient in forming a stable complex with the heterologous mouse factors. First, its expression is nearly abolished when it is cotranscribed with the mouse gene (lane 4), and second, even when preincubated in the S-100 extract under conditions where this promoter binds the limiting essential mouse transcription factors (data not shown), its complex is not sufficiently strong to prevent transcription of a subsequently added mouse promoter (lane 7). This result is consistent with previous studies in the mouse system, which found a close correlation between the transcriptional capacities of different templates and their efficiencies of complex formation (Tower et al. 1986).

The inefficient complex formation on the wild-type frog promoter contrasts sharply with the behavior of the frog template LS $-36 /-28(-5)$ bearing a half helical turn deletion. As shown in Figure 2, this template effectively outcompetes a cotranscribed wild-type frog promoter. Figure 7A extends these results by demonstrating that the stable complex formed on this frog spacing change template is able to withstand competition by a subsequently added mouse promoter (lane 8). In fact, when the frog spacing change promoter and the mouse promoter are simultaneously added to the extract, the template is efficiently transcribed and almost completely represses expression of the mouse template (Fig. 7A, lane 5). This result leads to the striking conclusion that with the mouse transcription factors, this heterologous frog rDNA promoter outcompetes the homologous mouse promoter.

Figure 7B shows a complementary sequential addition experiment in which mouse rDNA was preincubated in the extract. When challenged with a competitor mouse template, transcription from the first mouse template was favored, demonstrating a significant extent of complex formation (cf. lane 4 to lanes 2 and 3). Unexpectedly, however, when the mouse complex was challenged by subsequent addition of LS $-36 /-28(-5)$ DNA, transcription from the preincubated mouse template was severely reduced, whereas transcription from the added frog template occurred at control levels (cf. lane 5 to lanes 1 and 2). Therefore, the frog rDNA spacing change mutant binds the essential mouse rDNA transcription factors with such efficiency that it is able to dislodge them from their complex on the mouse rDNA promoter. Given the species specificity that is generally observed 


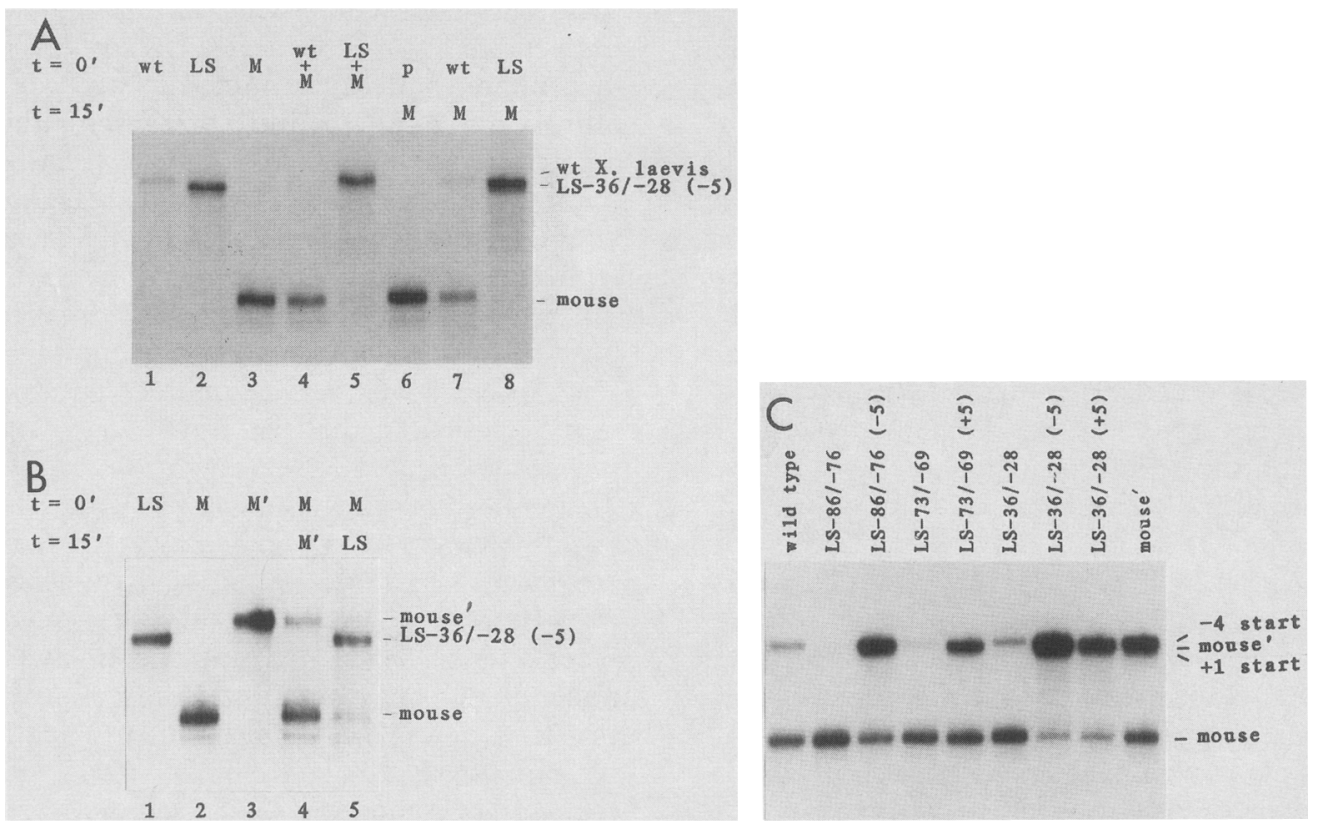

Figure 7. Transcriptional and stable binding capacities of frog rDNAs. $(A$ and $B)$ The wild-type Xenopus rDNA (wt), LS - 36/-28 $\mid-$ 5) (LS), and wild-type mouse rDNA, linearized either at residue $+292(\mathrm{M})$ or at residue $+445\left(\mathrm{M}^{\prime}\right)$, were preincubated alone, in combination, or following sequential addition to the extract, as indicated. (p) pBR322 deleted of the ClaI-SalI region. Nucleotides, including $\left[\alpha^{32}\right.$-P $]$ CTP, were added at $t=30 \mathrm{~min}$ to initiate a 45 -min synthesis period. Transcripts were directly identified by autoradiography following electrophoretic resolution. The experiment in $A$ used mouse plasmid $5^{\prime}$ 'Sal-Pvu. The experiment in $B$ used mouse plasmid $5^{\prime} \Delta-230$, and the various templates were added at equimolar concentration $(1.4 \mathrm{pmoles} / \mathrm{ml})$ and supplemented with pUC18 to achieve $4 \mu \mathrm{g} / \mathrm{ml}$, the concentration of the frog template. $|C|$ The indicated half helical turn spacing change mutants $\mid-5$ and +5 alterations) and their parental linker-scanning templates were transcribed in competition with an equimolar amount of mouse rDNA template, as in $(A$, lanes $1-5)$. Lane 1 used the wild-type frog rDNA; lane 9 shows the competition between two differently truncated mouse templates.

in rDNA transcription, this ability of the spacing change frog rDNA promoter to outcompete the mouse rDNA promoter for the binding of its homologous mouse factors is especially noteworthy.

From the data of Figure 7A and B, it could still have been argued that the great efficiency of the LS-36/ $-28(-5)$ template is attributable primarily to the precise nucleotide sequence created in the -30 region of this mutant promoter and not to the accompanying half helical turn spacing change. To address this possibility, we examined the capacity of the other half helical turn spacing change templates to compete with the mouse template in the mouse S-100 extract (using an assay analogous to that of Fig. 7A, lanes 4-5). As shown in Figure $7 \mathrm{C}$, all of these half helical turn insertion and deletion templates compete quite efficiently with the mouse rDNA template, whereas their parental constructs with wild-type spacing do not. Notably, LS $-36 /$ $-28(-5)$, LS $-36 /-28(+5)$, and LS $-86 /-76(-5)$ all outcompete the homologous mouse template, demonstrating that the very strong binding of the mouse factors is due largely to the spacing change and not primarily to the sequences at the site of the mutation.

We conclude that although the sequence information contained in the frog rDNA promoter is sufficient to bind the mouse transcription factors efficiently (specifically factor $\mathrm{D}$, which is responsible for template com- mitment; Tower et al. 1986), in the wild-type frog promoter, the essential upstream and core domains are on the wrong relative faces of the DNA helix to allow stable binding by the mouse factors. When the half helical turn spacing change mutants bring the core and upstream binding sites onto the correct (mouse-like) relative helix face, stable binding and efficient transcription can occur. Because all of the half helical turn spacing change mutants are efficient competitors (Fig. 7C), one can further conclude that stable binding of the essential mouse rDNA transcription factors can occur irrespective of the relative helical face of the sequences between the core and upstream promoter domains (at least those between residues -86 and -28 ).

\section{Discussion}

Extensive literature documents that polymerase I transcription factors generally are species specific and are not able to initiate synthesis on the rDNA promoter of even moderately closely related species (Grummt et al. 1982; Kohorn and Rae 1982; Learned and Tiian 1982; Mishima et al. 1982; Sollner-Webb and McKnight 1982; Culotta et al. 1987, and references therein). The most cited examples of this are the rDNA transcriptional incompatibility of mouse and human and of Drosophila melanogaster and Drosophila virilis, as well as of more 
distantly related species such as human and Xenopus. This apparently rapid evolution of polymerase I transcriptional machinery has been attributed to its effectively acting on only one gene, the many copies of which are kept homogeneous by unequal crossing-over; it contrasts sharply with the impressive ability of polymerase II and polymerase III factors to function across widely dissimilar species (e.g., Cortese et al. 1978; DeRobertis and Olson 1979; Chodosh et al. 1988). The one documented exception to this rule of rDNA transcriptional incompatibility among all but the most closely related species is the transcription of $X$. laevis rDNA by mouse cell factors (Wilkinson and Sollner-Webb 1982; Culotta et al. 1987). (Although water beetle rDNA has been shown to direct RNA synthesis in injected Xenopus oocytes, the specificity of initiation has not been investigated (Trendelenburg et al. 1978).]

This paper reports analysis of the heterologous Xenopus/mouse transcription. Using linker-scanning mutants of the $X$. laevis rRNA gene, we find that transcription by the mouse cell factors requires an upstream promoter domain (approximately residues -140 to -120 ) and a core promoter domain (approximately residues -30 to +10 ), with the intervening sequences showing considerably less importance (Fig. 1A). This result closely parallels that found in the homologous Xenopus transcription system (Windle and Sollner-Webb $1986 \mathrm{~b}$ ) and confirms that rDNA promoter recognition is quite conserved between mouse and Xenopus. The fact that the same mouse factors are involved in this heterologous transcription as in the homologous transcription of mouse rDNA (Culotta et al. 1987) further underscores this conservation.

The heterologous transcription of $X$. laevis rDNA in the mouse cell extract exhibits the interesting property of initiating at residue -4 (Figs. 1 and 3; Wilkinson and Sollner-Webb 1982). This is 4 bp upstream from the start site used in vivo and in homologous Xenopus transcription systems (Wilkinson and Sollner-Webb 1982). Frog rDNA thus has two potential start sites, at residue +1 and at residue -4 , on opposite sides of the DNA helix.

Investigating the effect of spacing change mutants in the heterologous transcription system, we found that all templates with insertions or deletions of $>2$ bp between the core and upstream promoter domains fail to initiate at the site used on the wild-type gene (Figs. 2-6). This demonstrates the requirement for precisely positioned promoter domains, as is the case in the homologous Xenopus system (Windle and Sollner-Webb 1986b). However, in the heterologous system, mutants that either increase or decrease the spacing by approximately a one-half helical turn show efficient initiation at position +1 . Thus, introducing a spacing change of approximately one-half helical turn anywhere in the $90 \mathrm{bp}$ between the core and upstream promoter domains causes the initiation site to shift approximately a one-half helical turn to the in vivo frog start site. The choice between potential initiation sites therefore is dictated by the upstream promoter domain and is transmitted over a $\geqslant 90$-bp region.
The upstream promoter domains of Xenopus and mouse rDNA do show a limited homology (Fig. 8A), with the relevant regions displaced by one and one-half helical turns. If an initiation factor binds to this common sequence and dictates the face of the helix on which the polymerase can initiate, then one would predict that mouse factors would initiate transcription on Xenopus rDNA on the opposite face of the helix, consistent with the observed -4 heterologous start site (Figs. 1 and 3). This scenario is also consistent with the observed shift of the initiation site to residue +1 in the $-1 / 2$, $+1 / 2$, and $+11 / 2$ helical turn spacing change mutants (Figs. 2-6).

Because the +1 initiation site is used quite efficiently in all of the approximately half helical turn spacing change mutants (Figs. 2, 4, and 6), and the one and onehalf helical turn spacing change mutant (Fig. 6), but not in the approximately full or approximately two turn helical spacing change mutants (Figs. 5 and 6), the face of the DNA helix on which initiation occurs is dictated by the stereo-specific alignment of the upstream promoter domain. On the other hand, the position of the upstream promoter domain does not precisely determine the initiation site, for $-5,-3,+3,+4,+5,+6,+7$, and +15 spacing change mutants all initiate at residue +1 , whereas $-1,0$, and +1 spacing change templates all initiate at residue -4 (Fig. 6B). Evidently, sequences near the initiation site, within the Xenopus core promoter domain, determine the precise position(s) at which transcription can potentially initiate (residues +1 and -4 ); the choice of the actual initiation site is then dictated by

A

X. 1aevis $(+1)$

Mouse $(+1)$

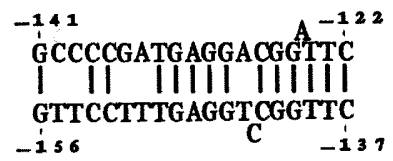

$B$

X. 1aevis $(-4)$

X. 1aevis (+1)

Mouse (+1)

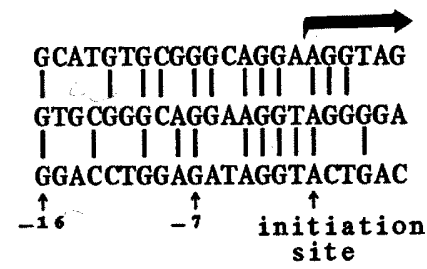

Figure 8. Sequence homology between the Xenopus and mouse rDNA promoters. (A) Potential homologies between the Xenopus and the mouse upstream domains are shown; numbering is relative to the natural initiation sites, +1 . $(B)$ The sequence of $X$. laevis rDNA, numbered relative to the in vivo initiation site, is shown in the middle line. The top line shows the $X$. laevis rDNA sequence, displaced to right by 4 nucleotides, so that the -4 initiation site used in the mouse cell extract lines up with the +1 initiation site of the middle line. There is a match of 11 of $15 \mathrm{bp}$ when the $X$. laevis promoter is aligned with itself in this manner. The sequence of the mouse rDNA initiation region, shown in the bottom line, aligned by its initiation site $(+1)$, exhibits a match of 8 of 11 bp with the $X$. laevis +1 site. Beyond this region, appreciable sequence homologies between the frog and mouse promoters (Sollner-Webb and Reeder 1979; Tower et al. 1986) are not observed. 
the helical face relative to the upstream promoter domain. It is possible that factor D binds to the upstream promoter domain (Culotta et al. 1987) and determines the face of the helix on which factor D can bind within the core promoter domain (Tower et al. 1986), thereby directing the choice of the initiation site.

Although the precise sequences in the core promoter domain that determine potential polymerase I initiation sites are not known, rDNA transcription almost universally begins at a purine that has $\mathrm{G}$ residues 7 and $16 \mathrm{nu}-$ cleotides upstream of it (Skinner et al. 1984; see also Tower et al. 1989). Consistent with this being an important determinant, the heterologous start site at position -4 conforms to this G/G/purine rule (Fig. 8B). However, this -4 start site shows additional homology with the sequences surrounding both the natural frog rDNA initiation site and the mouse rDNA initiation site, which could also be relevant (Fig. 8B). Other contacts within the core promoter domain land any within the interdomain region, including the major UBF-binding sites (Pikaard et al. 1989)] that are important for transcription, either must function irrespective of their helical face or must be repeated on the opposite side of the rDNA helix. It should also be noted that the core promoter region alone can direct these mouse factors to initiate on their homologous mouse rDNA (Yamomoto et al. 1984; Miller et al. 1985), suggesting an apparently duplicative function of the upstream and core promoter regions in choosing the initiation site. This almost assuredly results because the various promoter elements have evolved to act in a synergistic manner, and the in vitro system is able to function with just a subset of these natural interactions.

The requirement for stereo-specifically aligned domains of the $X$. laevis rDNA promoter can be compared to a number of other cases reported in the literature. The most cited examples are in prokaryotic systems, where action of Ara C protein (Dunn et al. 1984) or $\lambda$ repressor (Hochschild and Ptashne 1986) only occurs when pairs of binding sites are separated by whole helical repeats. The galactose and deo gene repressors appear to follow similar rules (Majumdar and Adhya 1984; Dandanell and Hammer 1985). In eukaryotic polymerase II systems, the thymidine kinase promoter elements do not appear to be affected by their stereo-specific alignment or by spacing alterations of up to $\sim 30 \mathrm{bp}$ (McKnight 1982), but these same elements in the SV40 early gene promoter and the ElB gene promoter exhibit a $10 \cdot n$ bp periodic spacing requirement that tolerates, respectively, considerable length alterations (Takahashi et al. 1986) or length alterations of one helical turn (Wu and Berk 1988). For RNA polymerase I transcription, spacing changes between promoter domains also appear to behave differently in various systems. As noted above, the Xenopus rDNA promoter is very strongly affected by half helical turn spacing changes between the core and upstream domains, both in the homologous frog system (Windle and Sollner-Webb 1986b) and in the heterologous mouse system, where a clear periodicity shows that the effects are stereo-specific (Fig. 6). In contrast, the human rDNA promoter has revealed no appreciable effect of spacing changes of up to $28 \mathrm{bp}$ between the core and upstream promoter domains (Haltiner et al. 1986; Jones et al. 1988|. This difference may be the result of the upstream promoter domain being essential in the former systems (Figs. 1, 2, 4; Windle and Sollner-Webb 1986a) but only stimulatory in the latter (Smale and Tiian 1985; Haltiner et al. 1986). In Acanthamoeba rDNA (which does not appear to have an upstream domain akin to metazoan rDNA; Kownin et al. 1985), transcription initiates a fixed distance from the factor binding site and exhibits limited tolerance for spacing changes (Kownin et al. 1987).

Another novel result of this study concerns the very active spacing change mutants of the frog rDNA promoter. Several of the approximately half helical turn insertion and deletion mutants not only use the natural frog initiation site at +1 but also direct considerably more transcription than the wild-type frog rDNA or their parental linker-scanning mutants. In fact, the templates LS $-36 /-28(-5)$, LS $-36 /-28(+5)$ and LS $-86 /$ $-76(-5)$ are so active in the mouse extract that they effectively compete out expression of mouse rDNA, the homologous template for these transcription factors (Fig. $7 \mathrm{~A}, \mathrm{Cl}$. Because the mouse rDNA stable preinitiation complex results from the binding of transcription factor D (Tower et al. 1986, 1989), the data of Figure 7 indicate that this binding is stronger on these frog promoters than on the homologous mouse rDNA promoter. Thus, these half helical turn spacing change frog promoters have become "mouse super promoters." These results suggest that rDNA transcriptional barriers between other pairs of species may also turn out to reflect only rather limited differences in their polymerase I transcriptional machinery.

\section{Materials and methods}

LS mutant and control plasmids

Construction of the linker-scanning and control genes was described previously (Windle and Sollner-Webb 1986b). The linker-scanning mutants were formed from $5^{\prime}$ and $3^{\prime}$ deletions and contain a 10-bp BamHI linker replacing a 10-bp $( \pm 1 \mathrm{bp})$ segment of rDNA in an otherwise complete promoter region (residues -245 to +115 or -317 to +115 ). (The numbers following LS identify the rDNA boundaries between which the mutations are located.) (LS $-154 /-143,-140 /-128,-115 /$ $-110,-106 /-97,-94 /-89,-65 /-58,-56 /-43,-49 /-43$, $-29 /-22,-18 /-9,-8 /+1,+7 /+10$, and $+14 /+23$ contain no spacing alterations: $L S-174 /-168,-165 /-157,-126 /$ $-120,-86 /-76$, and $-36 /-28$ are -1 spacing changes; and LS $-73 /-69$ is a +1 spacing change.) The spacing change derivatives of these templates result from insertions or deletions at the site of the number of residues, indicated in parenthesis, at the site of the linker relative to the wild-type template. Most of these were formed as described in Windle and Sollner-Webb (1986b). The spacing change derivatives not described previously were constructed as follows: LS $-86 /-76(-1)$ was cleaved at the BamHI site of the mutation, treated with $\mathrm{S} 1 \mathrm{nu}-$ clease, and religated to yield LS $-86 /-76(-5)$. LS -73 / $-69(+5)$ and $(+7)$ were made by cleaving LS $-73 /-69(+1)$ and $(+3)$, respectively, with $B a m H I$, filling in with Klenow poly- 
merase, and religating. For LS $-36 /-28(+5)$, LS $-36 /-28(-1)$ was cleaved at the BamHI site of the linker mutation, filled-in with Klenow enzyme in the presence of dGTP, treated with S1 nuclease, and ligated in the presence of 8-bp BglII linkers. LS $-36 /-28(+15)$ was made similarly, except the filling-in reaction was in the presence of $\mathrm{dG}, \mathrm{A}$ and TTP, and 12-bp EcoRI linkers were present in the ligation reaction. From LS-36/$28(+15)$, LS -36$)-28(+6),(+7)$, and $(+8)$ were made by cleaving at the EcoRI site of the linker and treating with 15 units of $\mathrm{S} 1$ nuclease for $15 \mathrm{~min}$ at $16^{\circ} \mathrm{C}$ and then for $15 \mathrm{~min}$ at $4^{\circ} \mathrm{C}$ followed by ligation. LS $-36 /-28|+19|$ was made by filling-in with Klenow polymerase in the presence of all four dNTPs, followed by ligation. The double mutant LS - 73/$69(-3),-18 /-9$ was made from a $P s t-B g I$ partial fragment $(-317$ to -62$)$ of LS $-73 /-69(-3)$ and the partial BgII-EcoRI fragment $(-61$ to end of the rDNA minigene), cloned in a PstIEcoRI pBR322 vector. The control gene contains the Xenopus rDNA promoter (from -245 to +13 ) linked to prokaryotic sequences so that its transcript can be detected by a different probe than that used for the linker-scanning mutants and the wild-type gene (the B gene of Windle and Sollner-Webb 1986b). The mouse templates $\mathrm{p} 5$ 'Sal-Pvu and $5^{\prime} \Delta-230$ were described previously (Miller and Sollner-Webb 1981; Henderson and Sollner-Webb 1986).

\section{Transcription in mouse S-100 extracts}

The S-100 extract was prepared from log-phase mouse L1210 tissue culture cells, as described previously (Miller and SollnerWebb 1981). Transcription reactions of $25 \mu \mathrm{l}$ contained $15 \mathrm{~mm}$ HEPES ( $\mathrm{pH} 7.9$ ), $10 \%$ glycerol, $1 \mathrm{~mm}$ dithiothreitol, $0.1 \mathrm{~mm}$ EDTA, $85 \mathrm{~mm} \mathrm{KCl}, 3.5 \mathrm{~mm} \mathrm{MgCl} 2,300 \mu \mathrm{g} / \mathrm{ml} \alpha$-amanitin, and 4-5 $\mu \mathrm{g} / \mathrm{ml}$ each of the linker-scanning mutant plasmid for wild-type plasmid) and the control plasmid. (For the experiments of Fig. $6 \mathrm{~A}$ and $7 \mathrm{C}$, the $\mathrm{KCl}$ and $\mathrm{MgCl}_{2}$ were 90 and $5 \mathrm{~mm}$, respectively; for the experiments of $\mathrm{Fig}$. $7 \mathrm{~A}, \mathrm{~B}$, the $\mathrm{KCl}$ and $\mathrm{MgCl}_{2}$ were 105 and $5 \mathrm{mM}$, respectively.) Linear templates were generally used because they resulted in somewhat more efficient transcription than supercoiled templates in these extracts. However, the same relative transcriptional efficiencies of the linker-scanning mutants were obtained with supercoiled templates (data not shown). The reactions were preincubated at $30^{\circ} \mathrm{C}$ for $15 \mathrm{~min}$. Transcription was initiated by addition of 500 $\mu \mathrm{M}$ each of ATP, GTP, UTP, and CTP and the reactions were incubated at $30^{\circ} \mathrm{C}$ for $60 \mathrm{~min}$. Transcription was terminated and RNA was isolated as described (Miller and Sollner-Webb 1981). For the experiments of Figures 1-6, the templates were linearized with PvuII, $2.9 \mathrm{~kb}$ downstream of the start site, and transcription was assessed by $\mathrm{S} 1$ nuclease analysis. For the experiments of Figure 7, the Xenopus rDNA plasmids were linearized with EcoRI, $457 \mathrm{bp}$ downstream from the start site, and following the two successive $15-\mathrm{min}$ preincubations, a $45-\mathrm{min}$ transcription period was initiated by addition of $500 \mu \mathrm{M}$ ATP, GTP, and UTP and $50 \mu \mathrm{M}$ CTP containing $3 \mu \mathrm{Ci}[\alpha-32 \mathrm{P}] \mathrm{CTP}$ per reaction. The resultant radioactive transcripts were resolved by electrophoresis and detected by autoradiography. The mouse templates for Figure 7 were linearized with $P$ vuII at residue + 292 or with TthI 445 bp downstream from the start site. Dinucleotide analysis was performed as described previously (Wilkinson et al. 1983).

\section{S1 nuclease analysis}

For S1 nuclease analysis, one-half to one-fifth of the RNA from an in vitro reaction was hybridized to 0.01 pmole of probe, and the hybrids were treated with S1 nuclease and analyzed as described (Windle and Sollner-Webb 1986a). The "Hinf" DNA probe (for assay of transcripts of the linker-scanning and wildtype genes) and the " $\mathrm{B}$ " probe (for assay of the control transcript) have been described previously (Windle and SollnerWebb 1986a).

\section{Acknowledgments}

We thank Ms. Maria Ysern for technical assistance and Ms. Margaret Minnick for help in preparing the manuscript. This work was supported by grant GM27720 from the National Institutes of Health (to B.S.-W.) and postdoctoral fellowship PF-2831 from the American Cancer Society (to L.P.).

\section{References}

Chodosh, L.A., J. Olesen, S. Hahn, A.S. Baldwin, L. Guarente, and P.A. Sharp. 1988. A yeast and a human CCAAT-binding protein have heterologous subunits that are functionally interchangeable. Cell 53: 25-35.

Cizewski, V.C. and B. Sollner-Webb. 1983. A stable transcription complex directs mouse ribosomal RNA synthesis by RNA polymerase I. Nucleic Acids Res. 11: 7043-7056.

Cortese, R., D. Melton, T. Tranquilla, and J.D. Smith. 1978. Cloning of nematode tRNA genes and their expression in the frog oocyte. Nucleic Acids Res. 5: 4593-4604.

Culotta, V.C., J.K. Wilkinson, and B. Sollner-Webb. 1987. Mouse and frog violate the paradigm of species-specific transcription of ribosomal RNA genes. Proc. Natl. Acad. Sci 84: 7498-7502.

Dandanell, G. and K. Hammer. 1985. Two operator sites separated by 599 base pairs are required for deoR repression of the deo operon of Escherichia coli. EMBO J. 4: 3333-3338.

DeRobertis, E. and M. Olson. 1979. Transcription and processing of yeast tyrosine tRNA genes microinjected into frog oocytes. Nature 278: 137-143.

Dunn, T.M., S. Hahn, S. Ogden, and R.F. Schleif. 1984. An operator at -280 base pairs that is required for repression of araBAD operson promoter: Addition of DNA helical turns between the operator and promoter cyclically hinders repression. Proc. Nat1. Acad. Sci. 81: 5017-5020.

Grummt, I. 1982. Nucleotide sequence requirements for specific initiation of transcription by RNA polymerase I. Proc. Natl. Acad. Sci. 79: 6908-6911.

Grummt, I., E. Roth, and M.R. Paule. 1982. Ribosomal RNA transcription in vitro is species specific. Nature 296: 173174.

Haltiner, M.M., S.T. Smale, and R. Tjian. 1986. Two distant promoter elements in the human rDNA gene identified by linker scanning mutagenesis. Mol. Cell. Biol. 6: 227-235.

Henderson, S. and B. Sollner-Webb. 1986. A transcriptional terminator is a novel element of the promoter of the mouse ribosomal RNA gene. Cell 47: 891-900.

Hochschild, A. and M. Ptashne. 1986. Cooperative binding of $\lambda$ repressors to sites separated by integral turns of the DNA helix. Cell 44: 681-687.

Iida, C.T., P. Kownin, and M.R. Paule. 1985. Ribosomal RNA transcription: Proteins and DNA sequences involved in preinitiation complex formation. Proc. Natl. Acad. Sci. 82: $1668-1672$.

Jones, M.H., R.M. Learned, and R. Tiian. 1988. Analysis of clustered point mutations in the human ribosomal RNA gene promoter by transient expression in vivo. Proc. Natl. Acad. Sci. 85: 669-673.

Kohorn, B.D. and P.M.M. Rae. 1982. Accurate transcription of truncated ribosomal DNA templates in a Drosophila cellfree system. Proc. Natl. Acad. Sci. 79: 1501-1505. 
Kownin, P., C.T. Iida, S. Brown-Shimer, and M.P. Paule. 1985. The ribosomal RNA promoter of Acanthamoeba castellanii determined by transcription in a cell-free system. Nucleic Acids Res. 13: 6237-6248.

Kownin, P., E. Bateman, and M.R. Paule. 1987. Eukaryotic RNA polymerase I promoter binding is directed by protein contacts with transcription initiation factor and is DNA sequence-independent. Cell 50: 693-699.

Learned, R.M. and R. Tjian. 1982. In vitro transcription of human ribosomal RNA genes by RNA polymerase I. J. Mol. Appl. Genet. 1: 575-584.

Learned, R.M., S. Smale, M.M. Haltiner, and R. Tiian. 1983. Regulation of human rRNA transcription. Proc. Natl. Acad. Sci. 80: 3558-3562.

Lopata, M., D. Cleveland, and B. Sollner-Webb. 1986. Polymerase specificity of mRNA processing and enhancer action. Proc. Natl. Acad. Sci. 83: 6677-6681.

Majumdar, A. and B. Adhya. 1984. Demonstration of two operator elements in gal: In vitro repressor binding sites. Proc. Natl. Acad. Sci. 81: 6100-6104.

Maxam, A. and W. Gilbert. 1980. Sequencing end-labelled DNA with base-specific chemcial cleavages. Methods Enzymol. 65: 499-560.

McKnight, S.L. 1982. Functional relationships between transcriptional control signals of the thymidine kinase gene of herpes simplex virus. Cell 31: 355-365.

Miesfeld, R. and N. Arnheim. 1984. Species-specific rDNA transcription is due to promoter-specific binding factors. Mol. Cell. Biol. 4: 221-227.

Miller, K.G. and B. Sollner-Webb. 1981. Transcription of mouse rRNA genes by RNA polymerase I: In vitro and in vivo initiation and processing sites. Cell 27: 165-174.

Miller, K.G., J. Tower, and B. Sollner-Webb. 1985. A complex control region of the mouse rRNA gene directs accurate initiation by RNA polymerase I. Mol. Cell. Biol. 5: 554-562.

Mishima, Y., I. Financsek, R. Kominami, and M. Muramatsu. 1982. Fractionation and reconstitution of factors required for accurate transcription of mammalian ribosomal RNA genes: Identification of a species-dependent initiation factor. Nucleic Acids Res. 10: 6659-6670.

Moss, T. 1982. Transcription of cloned Xenopus laevis ribosomal DNA microinjected into Xenopus oocytes, and the identification of an RNA polymerase I promoter. Cell 30: $835-842$.

Pikaard, C. S., McStay, B., M.C. Schulta, S.P. Bell, and R.H. Reeder. 1989. The Xenopus ribosomal gene enhancers bind an essential polymerase I transcription factor, xUBF. Genes Dev. (in press).

Reeder, R.H., D. Pennock, B. McStay, J. Roan, E. Tolentino, and P. Walker. 1987. Linker scanner mutagenesis of the Xenopus laevis ribosomal gene promoter. Nucleic Acids Res. 15: $7429-7441$.

Skinner, I., A. Ohrlein, and I. Grummt. 1984. In vitro mutagenesis and transcriptional analysis of a mouse ribosomal promoter element. Proc. Natl. Acad. Sci. 81: 2137-2141.

Smale, S.T. and R. Tiian. 1985. Transcription of herpes simplex virus tk sequences under the control of wild-type and mutant human RNA polymerase I promoters. Mol. Cell. Biol. 5: $352-362$.

Sollner-Webb, B. and R. Reeder. 1979. The nucleotide sequence of the initiation and termination sites for rRNA transcription in Xenopus laevis. Cell 18: 485-499.

Sollner-Webb, B. and S. McKnight. 1982. Accurate transcription of cloned Xenopus ribosomal RNA genes. Nucleic Acids Res. 10: 3391-3405.
Sollner-Webb, B., J. Wilkinson, J. Roan, and R. Reeder. 1983. Nested control regions promote Xenopus rRNA synthesis by RNA polymerase I. Cell 35: 199-206.

Takahashi, K., M. Vigneron, H. Matthes, A. Wildeman, M. Zenke, and P. Chambon. 1986. Requirement of stereo-specific alignments for initiation from the simian virus 40 early promoter. Nature 319: 121-126.

Tower, J., V. Culotta, and B. Sollner-Webb. 1986. The factors and nucleotide sequences that direct rDNA transcription and their relationships to the stable transcription complex. Mol. Cell. Biol. 6: 3451-3462.

Tower, J., S.L. Henderson, K.M. Dougherty, P.J. Wejksnora, and B. Sollner-Webb. 1989. An RNA polymerase I promoter located in the $\mathrm{CHO}$ and mouse ribosomal DNA spacers: Functional analysis and factor sequence requirements. Mol. Cell. Biol. 9: 1513-1525.

Trendelenburg, M.F., H. Zentgraf, W.W. Franke, and J.B. Gurdon. 1978. Transcription patterns of amplified Dytiscus genes coding for ribosomal RNA after injection into Xenopus oocyte nuclei. Proc. Natl. Acad. Sci. 75:37913795.

Vance, V.B., E.A. Thompson, and L.H. Bowman. 1985. Transfection of mouse ribosomal DNA into rat cells. Faithful transcription and processing. Nucleic Acids Res. 13: 74997513.

Wilkinson, J. and B. Sollner-Webb. 1982. Transcription of Xenopus ribosomal RNA genes by RNA polymerase I in vitro. J. Biol. Chem. 257: 14375-14383.

Wilkinson, J., K. Miller, and B. Sollner-Webb. 1983. Dinucleotide primers facilitate convenient identification of the mouse ribosomal DNA transcription initiation site. /. Biol. Chem. 258: 13919-13928.

Windle, J.J. and B. Sollner-Webb. 1986a. Upstream domains of the Xenopus laevis rDNA promoter are revealed in microinjected oocytes. Mol. Cell. Biol. 6: 1228-1234.

- 1986b. Two distant and precisely positioned domains promote transcription of Xenopus laevis rRNA genes: Analysis with linker-scanning mutants. Mol. Cell. Biol. 6: 45854593.

Wu, L. and A. Berk. 1988. Constraints on spacing between transcription factor binding sites in a simple adenovirus promoter. Genes Dev. 2: 403-411.

Yamamoto, O.N., N. Takakusa, Y. Mishima, R. Kominami, and M. Muramatsu. 1984. Determination of the promoter region of mouse rRNA gene by an in vitro transcription system. Proc. Natl. Acad. Sci. 81: 299-303. 


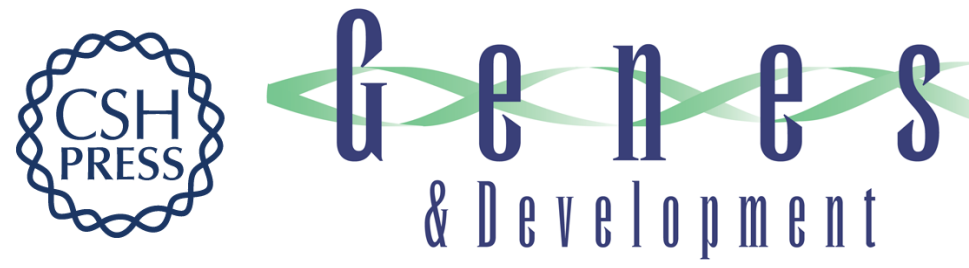

\section{Half helical turn spacing changes convert a frog into a mouse rDNA promoter: a distant upstream domain determines the helix face of the initiation site.}

L K Pape, J J Windle and B Sollner-Webb

Genes Dev. 1990, 4:

Access the most recent version at doi:10.1101/gad.4.1.52

References This article cites 45 articles, 23 of which can be accessed free at: http://genesdev.cshlp.org/content/4/1/52.full.html\#ref-list-1

License

Email Alerting Receive free email alerts when new articles cite this article - sign up in the box at the top Service right corner of the article or click here.



Focused on your science. 